\title{
Second-Generation Trabecular Micro-Bypass (iStent inject) with Cataract Surgery in Eyes with Normal- Tension Glaucoma: One-Year Outcomes of a Multi- Centre Study
}

\author{
Ali Salimi (D) - Colin Clement · Michael Shiu $\cdot$ Paul Harasymowycz
}

Received: May 5, 2020 / Published online: July 1, 2020

(C) The Author(s) 2020

\section{ABSTRACT}

Purpose: The efficacy and safety of the trabecular micro-bypass stents (iStent and iStent inject) have been well documented in various open-angle glaucoma subtypes. However, their

Digital Features To view digital features for this article go to https://doi.org/10.6084/m9.figshare.12389639.

\author{
A. Salimi \\ Department of Ophthalmology, Faculty of \\ Medicine, McGill University, Montreal, QC, Canada \\ C. Clement \\ Eye Associates, Sydney, NSW, Australia \\ C. Clement \\ Fairfield Eye Surgery, Fairfield, NSW, Australia \\ C. Clement \\ Glaucoma Unit, Sydney Eye Hospital, Sydney, NSW, \\ Australia \\ C. Clement \\ Discipline of Ophthalmology, The University of \\ Sydney, Sydney, NSW, Australia \\ M. Shiu \\ Laser Sight Centre, Melbourne, VIC, Australia \\ P. Harasymowycz ( $\square)$ \\ Montreal Glaucoma Institute, Montreal, QC, \\ Canada \\ e-mail: pavloh@igmtl.com \\ P. Harasymowycz \\ Department of Ophthalmology, University of \\ Montreal, Montreal, QC, Canada
}

outcomes remain understudied in normal-tension glaucoma (NTG). The present study aimed to assess the 1-year outcomes related to the implantation of two second-generation trabecular micro-bypass stents (iStent inject) concomitant with cataract surgery (CE-TMS), exclusively in eyes with NTG.

Methods: This multi-center, consecutive case series included eyes with cataract and normaltension glaucoma that underwent CE-TMS to reduce intraocular pressure or glaucoma medication use. The 12-month efficacy measures included change in average intraocular pressure (IOP) and medication burden. Safety included change in best-corrected visual acuity, cup-todisc ratio, visual field mean-deviation and retinal nerve fiber layer thickness. Intra- or postoperative adverse events were noted.

Results: A total of 62 eyes with mild-to-severe NTG and average preoperative IOP of $15.82 \pm 2.94 \mathrm{mmHg}$ on $1.50 \pm 1.28$ glaucoma medications were included. Postoperatively, IOP declined by $22 \%$ from $15.82 \pm 2.94 \mathrm{mmHg}$ to $12.32 \pm 2.58 \quad(p<0.001), \quad$ all eyes had IOP $\leq 18 \mathrm{mmHg}$ (versus $74 \%$ preoperatively), and half had IOP $\leq 12 \mathrm{mmHg}$ (versus $15 \%$ preoperatively). Medication burden decreased by $70 \%$ from $1.50 \pm 1.28$ to $0.45 \pm 0.86(p<0.001)$, and $73 \%$ of the eyes were medication-free (versus $23 \%$ preoperatively). Safety was favorable, with no evidence of sight-threatening adverse events. Conclusion: Implantation of iStent inject (two second-generation trabecular micro-bypass 
stents) combined with cataract surgery is efficacious in reducing IOP and medication burden with a favorable safety profile in eyes with mildto-severe NTG.

Keywords: Cataract; Glaucoma; iStent inject; Micro-invasive glaucoma surgery (MIGS); Normal-tension glaucoma; NTG; Stent; Trabecular micro-bypass

\section{Key Summary Points}

\section{Why carry out this study?}

Numerous studies have established the safety and effectiveness of iStent inject trabecular micro-bypass implantation in combination with cataract surgery in eyes with different types of glaucoma. However, their outcomes remain understudied in normal-tension glaucoma (NTG).

The present study aims to assess the 1-year outcomes of iStent inject implantation with cataract surgery exclusively in eyes with NTG.

\section{What was learned from the study?}

Intraocular pressure (IOP) at 12 months was significantly lower than preoperative IOP $(12.3 \pm 2.6 \mathrm{mmHg}$ versus $15.8 \pm 2.9 \mathrm{mmHg}$, respectively; $p<0.001)$. At 12 months, all eyes achieved IOP $\leq 18 \mathrm{mmHg}$ (versus $74 \%$ preoperatively), $92 \%$ of eyes achieved IOP $\leq 15 \mathrm{mmHg}$ (versus $45 \%$ preoperatively), and half (50\%) of the eyes achieved IOP $\leq 12 \mathrm{mmHg}$ (versus $15 \%$ preoperatively).

Medication burden at 12 months was significantly reduced from preoperative $(0.45 \pm 0.86$ medications versus $1.50 \pm 1.28$ medications, respectively; $p<0.001)$. All eyes either maintained or decreased topical medications from baseline, with $66 \%$ of eyes eliminating $\geq 1$ medication and $29 \%$ of eyes eliminating $\geq 2$ medications from their preoperative regimen.
Implantation of two second-generation trabecular micro-bypasses combined with cataract surgery is efficacious in reducing IOP and medication burden with a favorable safety profile in eyes with mildto-severe NTG.

\section{INTRODUCTION}

Glaucoma is the leading cause of irreversible blindness worldwide and is expected to affect 111 million people by 2040 [1]. Current approaches to glaucoma treatment are focused on decreasing intraocular pressure (IOP)-the only modifiable risk factor proven to be associated with disease progression [2, 3]. Normaltension glaucoma (NTG) is a variant of openangle glaucoma (OAG) and is characterized by glaucomatous damage despite having IOP values constantly $<21 \mathrm{mmHg}$ [4]. The elevated IOP has often been considered an integral diagnostic feature of glaucoma, making detection of NTG particularly challenging; thus normotensive NTG patients frequently have been underdiagnosed and under-treated [5]. Despite normal IOP values, the Collaborative Normal Tension Glaucoma Study (CNTGS) evidenced a strong association between IOP lowering and deceleration in disease progression $[3,6]$. It was concluded that IOP reduction, whether medically or surgically, remains the most important component of NTG treatment $[3,6]$.

Micro-invasive glaucoma surgery (MIGS) is a growing area of surgical glaucoma treatment options that offers IOP-reducing efficacy alongside a superior safety profile compared to traditional glaucoma surgeries [7]. These ab-interno, tissue-sparing MIGS procedures lead to quicker visual recovery, spare conjunctival tissue for future glaucoma surgeries [8] and can be undertaken as a standalone procedure or combined with cataract or other MIGS surgeries $[9,10]$. The introduction of MIGS has stimulated a more proactive, surgical approach in treating mild-to-moderate OAG [11]. Recently, MIGS procedures have been used with or without the traditional early interventions of 
medication and/or laser trabeculoplasty [12]. A surgical intervention avoids the limitations associated with the glaucoma medications such as low patient adherence, side effects, ocular surface damage and costs [13-17], while also limiting IOP fluctuations compared to medical therapy [18].

iStent trabecular micro-bypass (Glaukos Corp., San Clemente, CA, USA) was the first MIGS device to receive the US Food and Drug Association (FDA) approval for treatment of mild-to-moderate OAG. Recently, the second-generation iStent inject trabecular micro-bypass (iStent inject) was approved for the same. Both devices are designed to restore natural physiologic outflow by creating a patent bypass through the trabecular meshwork into Schlemm's canal for aqueous humor to exit the anterior chamber. In addition to some fundamental differences in design, iStent inject has the advantage of coming pre-loaded with two stents, which allows placement in separate regions of the trabecular meshwork to access a larger number of collector channels. Additionally, the stents contain a central lumen with four side outlets allowing a multidirectional aqueous outflow.

iStent inject has been extensively studied, and its efficacy and safety have been established in primary OAG, secondary OAG and ocular hypertension [19-27]; however, to our knowledge, no study has examined its use exclusively in NTG. Here, we aimed to evaluate the 1-year outcomes of iStent inject with concomitant cataract surgery in patients with mild-to-severe NTG.

\section{METHODS}

\section{Participants and Design}

This retrospective multicenter consecutive case series comprised eyes with normal-tension glaucoma and cataract that underwent cataract extraction with concomitant implantation of two second-generation trabecular micro-bypass stents (iStent inject) (CE-TMS). All procedures were performed between 2016 and 2019, by three surgeons (P.H, C.C, M.S) at three separate ophthalmology centers. The inclusion criteria included: age $>18$ years; clinical diagnosis of
NTG; presence of cataract and the need for reduction of IOP or glaucoma medication in that eye; a minimum of 12 months postoperative follow-up data. NTG was defined as a patient having no history of maximal untreated IOP $>21 \mathrm{mmHg}$ and no history of trauma. Exclusion comprised the inability to visualize nasal trabecular meshwork, elevated episcleral venous pressure and ocular inflammation. At the time of this study, iStent inject was indicated for mild-to-moderate open-angle glaucoma; however, to increase the generalizability of our findings we did not exclude any eyes based on glaucoma severity or history of prior glaucoma surgery.

The study was approved by the Ethics Committee of the Hôpital Maisonneuve-Rosemont in Montreal, Canada. All procedures performed were in accordance with the ethical standards of the institutional research committee and with the 1964 Helsinki Declaration and its later amendments or comparable ethical standards. Voluntary informed consent was obtained from all participants before the surgery.

\section{Surgical Technique}

To ensure maximum proximity of the stent to the collector channel ostia two areas, located at least two clock hours apart in the nasal angle with greater trabecular pigmentation [28] or with evidence of focal blood reflux [8], were identified and marked at the corneal limbus [29]. Following the instillation of topical anesthesia, a small clear corneal incision was made temporally through which the iStent inject injector was inserted. Following visualization of the angle using gonioscopy, the two stents were implanted into the trabecular meshwork next to the collector channel markings. Through the same corneal incision, P.H. performed the phacoemulsification prior to the insertion of iStent $(n=26)$ while C.C. and M.S. performed this step following the iStent insertion $(n=36)$.

The glaucoma medication regimen was adjusted, case by case, as per the surgeon's discretion according to the severity of the disease and the preoperative IOP. The standard postoperative regimen for P.H. comprised one oral acetazolamide $500 \mathrm{mg}$ on the first evening, 1 
week of topical moxifloxacin (three times per day), 1 month of topical nepafenac (three times per day) and topical loteprednol, which was tapered from four times per day over 2 weeks. The regimen for C.C. and M.S. included 2 weeks of Chloramphenicol, Ketorolac and Maxidex/ Prednefrin Forte, each used four times per day followed by 2 weeks of Ketorolac and of Maxidex/Prednefrin used twice per day.

\section{Outcome Measures and Data Analysis}

Demographic, baseline and postoperative clinical data were extracted from patients' charts. Efficacy measures included changes in IOP (measured using the Goldman applanation tonometry) and anti-glaucoma medications (scored according to the number of active pharmacologic classes) [30, 31]. Safety comprised intra- or postoperative adverse events, best-corrected visual acuity (BCVA), cup-to-disc ratio (CDR), visual field mean deviation (VF-MD), increase in the number of glaucoma medications or secondary glaucoma interventions for uncontrolled IOP. Disease severity was classified according to the HodappAnderson-Parrish visual field criteria with VFMD no worse than $-6 \mathrm{~dB}$ classified as mild, between -6 and $-12 \mathrm{~dB}$ as moderate and worse than $-12 \mathrm{~dB}$ as severe [32].

Generalized linear models (GLM) evaluated the 1-year outcomes. Proportional analyses of eyes with IOP $\leq 18 \mathrm{mmHg}, \leq 15 \mathrm{mmHg}$ and $\leq 12 \mathrm{mmHg}$, and with medication reduction $\geq 0, \geq 1$ and $\geq 2$ medications, were performed and compared to the preoperative values. To ensure continuity of data, BCVA scores were converted to the logarithm of minimal angle of resolution (LogMAR). All statistical analyses were performed using SPSS 25.0 (IBM, NY, USA) with significance set at $p<0.05$.

\section{RESULTS}

\section{Demographics}

A total of 62 phakic eyes of 42 patients, with an average age of $71.9 \pm 7.4$ years and baseline IOP
Table 1 Demographic and preoperative ocular characteristics

\begin{tabular}{ll}
\hline Variable & $\begin{array}{l}\boldsymbol{N}=\mathbf{6 2} \text { eyes of 42 } \\
\text { subjects }\end{array}$ \\
\hline Age at time of surgery (years) & $71.92 \pm 7.45$ \\
Gender (male: female) & $24: 38$ \\
Eye (OD: OS) & $29: 33$ \\
$\begin{array}{l}\text { History of selective laser } \\
\text { trabeculoplasty \% ( } n)\end{array}$ & $23 \%(14)$ \\
Central corneal thickness $(\mu \mathrm{m})$ & $542.64 \pm 39.76$ \\
$\begin{array}{l}\text { Intraocular pressure (mmHg) } \\
\text { Glaucoma medications }\end{array}$ & $15.82 \pm 2.94$ \\
Corrected distance visual acuity & $0.17 \pm 0.21$ \\
$\quad$ logMar) & \\
Cup-to-disc ratio & $0.71 \pm 0.14$ \\
Visual field-mean deviation $(\mathrm{dB})$ & $-5.62 \pm 5.16$ \\
$\begin{array}{l}\text { Visual field-pattern standard } \\
\text { deviation (dB) }\end{array}$ & $4.76 \pm 2.98$ \\
$\begin{array}{l}\text { Retinal nerve fiber layer thickness } \\
\text { ( } \mu \text { m) }\end{array}$ & $76.94 \pm 13.09$ \\
\hline
\end{tabular}

Mean \pm standard deviations are presented, where applicable

of $15.82 \pm 2.94 \mathrm{mmHg}$ on $1.50 \pm 1.28$ glaucoma medications, were included. Most of the eyes (89\%) had mild-to-moderate NTG, and the remaining $11 \%$ had severe disease. Over three quarters $(77 \%)$ of the eyes had no prior glaucoma interventions, and the remaining 23\% had previously undergone a selective laser trabeculoplasty (SLT) procedure. None of the patients had previously undergone incisional glaucoma surgery. Demographics and baseline characteristics of the eyes are presented in Table 1.

\section{Efficacy}

Following CE-TMS, both IOP and medication use decreased significantly and consistently throughout the 12-month follow-up. Average IOP decreased by $22 \%$ from $15.8 \pm 2.9 \mathrm{mmHg}$ 


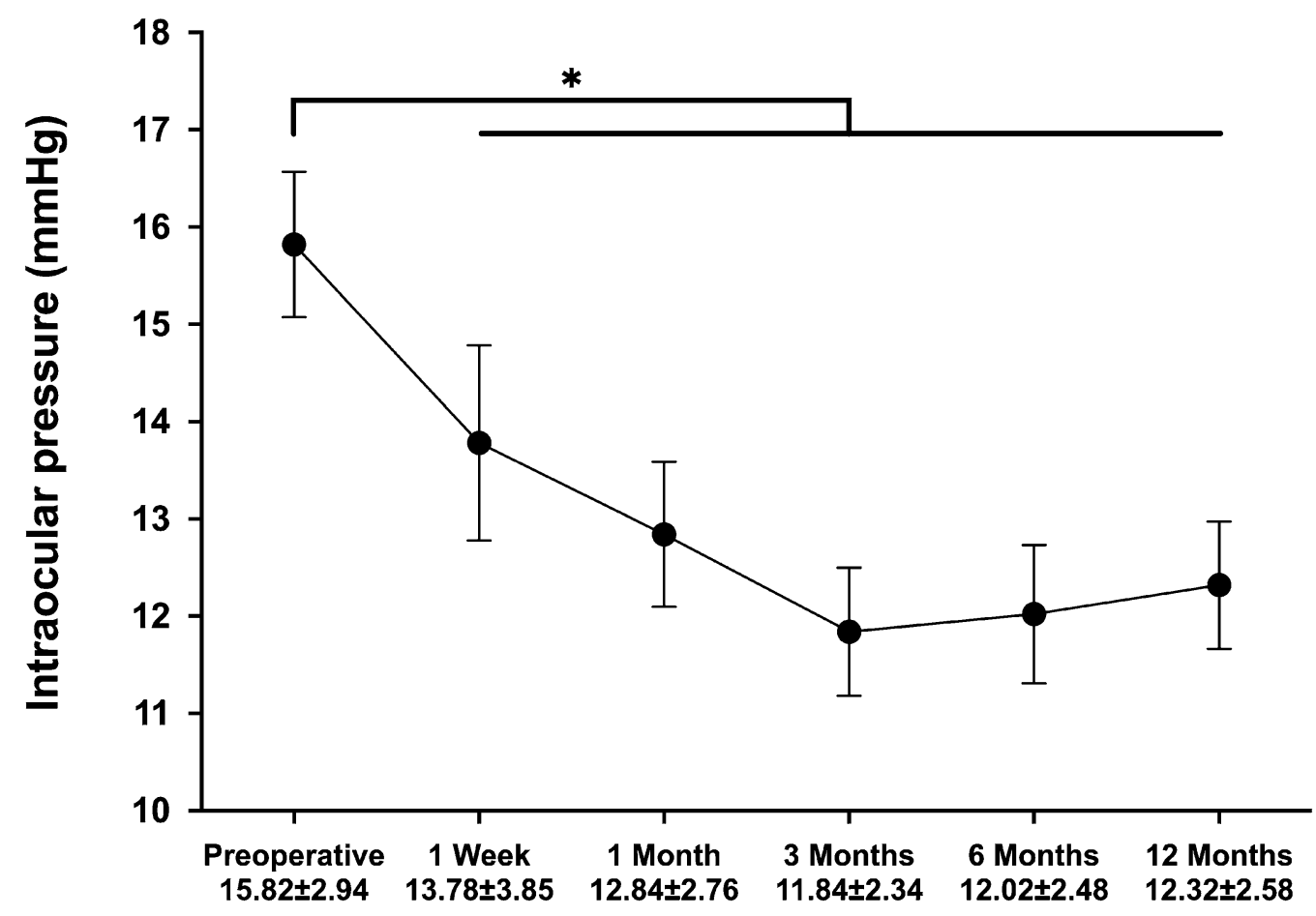

Fig. 1 Postoperative change in intraocular pressure. Following cataract surgery combined with implantation of iStent inject, the average intraocular pressure significantly decreased and was maintained throughout follow-up

preoperatively to $12.3 \pm 2.6 \mathrm{mmHg}$ at 12-month follow-up ( $p<0.001$, Fig. 1; Table 2). As presented in Fig. 2, proportional analyses $(p<0.001) .{ }^{*}$ Denotes statistical significance at $p<0.05$, and the error bars represent $95 \%$ confidence intervals. Mean \pm standard deviations are presented

highlighted a higher proportion of eyes with lower IOP at follow-up: all eyes had IOP $\leq 18 \mathrm{mmHg}$ (versus $74 \%$ preoperatively),

Table 2 Twelve-month outcomes in efficacy and safety measures ( $N=62$ eyes of 42 subjects)

\begin{tabular}{lccc}
\hline Variable & Baseline & 12-Month follow-up & $p$-value \\
\hline Intraocular pressure (mmHg) & $15.82 \pm 2.94[9-20]$ & $12.32 \pm 2.58[8-18]$ & $<0.001^{*}$ \\
Glaucoma medications & $1.50 \pm 1.28[0-5]$ & $0.45 \pm 0.86[0-4]$ & $<0.001^{*}$ \\
$\begin{array}{l}\text { Corrected distance visual acuity } \\
\quad(\text { logMar) }\end{array}$ & $0.17 \pm 0.21[0.0-1.0]$ & $0.04 \pm 0.09[0.0-0.4]$ & $<0.001^{*}$ \\
Cup-to-disc ratio & $0.71 \pm 0.14[0.40-0.95]$ & $0.73 \pm 0.12[0.40-0.95]$ & 0.527 \\
$\begin{array}{l}\text { Visual field-mean deviation }(\mathrm{dB}) \\
\text { Visual field-pattern standard }\end{array} \quad-5.62 \pm 5.16[-29.0$ to -0.5$]$ & $-5.97 \pm 5.00[-29.0$ to -0.5$]$ & 0.602 \\
$\quad$ deviation $(\mathrm{dB})$ & $4.76 \pm 2.98[2-13]$ & $4.38 \pm 2.74[1-12]$ & 0.483 \\
Retinal nerve fiber layer thickness & $76.94 \pm 13.09[52-114]$ & $77.75 \pm 14.14[50-114]$ & 0.777 \\
$\quad(\mu \mathrm{m})$ & & & \\
\hline
\end{tabular}

Mean \pm standard deviations are presented and statistically compared

${ }^{*}$ Denotes statistical significance

[] Represents range 


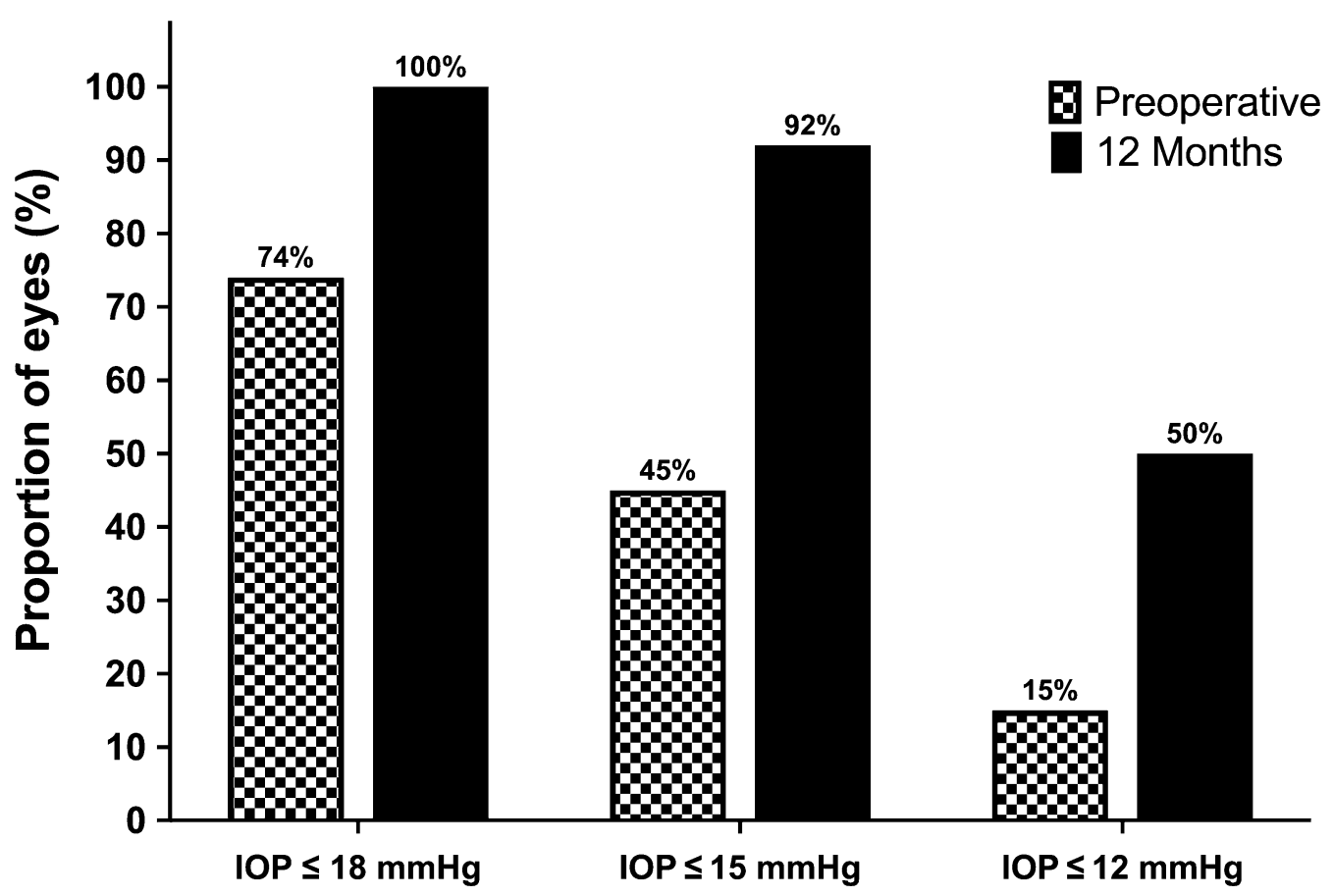

Fig. 2 Percentages of eyes with IOP $\leq 18, \leq 15$ and $\leq 12 \mathrm{mmHg}$ preoperatively and at 1-year follow-up. Solid and checked bars represent preoperative and 1-year follow-up data, respectively; IOP intraocular pressure

$92 \%$ had $\mathrm{IOP} \leq 15 \mathrm{mmHg}$ (versus $45 \%$ preoperatively), and $50 \%$ of the eyes had IOP $\leq 12 \mathrm{mmHg}$ (versus $15 \%$ preoperatively). The preoperative and the 12-month postoperative IOPs in each patient are presented in Fig. 3.

The average number of glaucoma medications decreased by $70 \%$ from $1.50 \pm 1.28$ medications preoperatively to $0.45 \pm 0.86$ medications at follow-up ( $p<0.001$, Fig. 4; Table 2). At 1-year postoperatively, medication use decreased by at least one medication among $66 \%$ and by at least two medications among $29 \%$. Furthermore, $73 \%$ of the eyes were medication-free (versus $23 \%$ preoperatively) and only $13 \%$ were on two or more glaucoma medications (versus 39\%, Fig. 5). No patient required an increase in the number of medications following surgery.

At the time of this study, iStent inject was approved for OAG with mild-to-moderate severities. Therefore, we performed a subgroup analysis excluding the severe cases. A total of 55 mild and moderate NTG eyes with an average age of $71.4 \pm 7.7$ were included. Preoperative IOP was $15.90 \pm 2.93$; the mean number of glaucoma medications was $1.44 \pm 1.21$. The primary outcomes were similar to those of the overall cohort. IOP decreased by $21.3 \%$ to $12.51 \pm 2.52(p<0.001)$, and the number of glaucoma medications decreased by $75 \%$ to $0.36 \pm 0.78(p<0.001)$. These outcomes were not significantly different from those of the severe cases either $(p>0.05)$.

\section{Safety}

All eyes successfully underwent CE-TMS with no intraoperative complications. BCVA improved by six letters of acuity $(p<0.001)$. No evidence of disease progression was noted within the first postoperative year, with stable CDR $(p=0.527)$, VF-MD $(p=0.602)$, VF$\operatorname{PSD}(p=0.483)$ and RNFL thickness $(p=0.777)$.

Postoperative adverse events were rare and self-limiting; IOP spike (IOP increase by $10 \mathrm{mmHg}$ or $\geq 50 \%$ above baseline) $[26,33]$ occurred in four eyes $(6.5 \%)$, and transient corneal edema was evidenced in five eyes $(8.1 \%)$, all within the first postoperative week. No evidence of sight-threatening adverse 


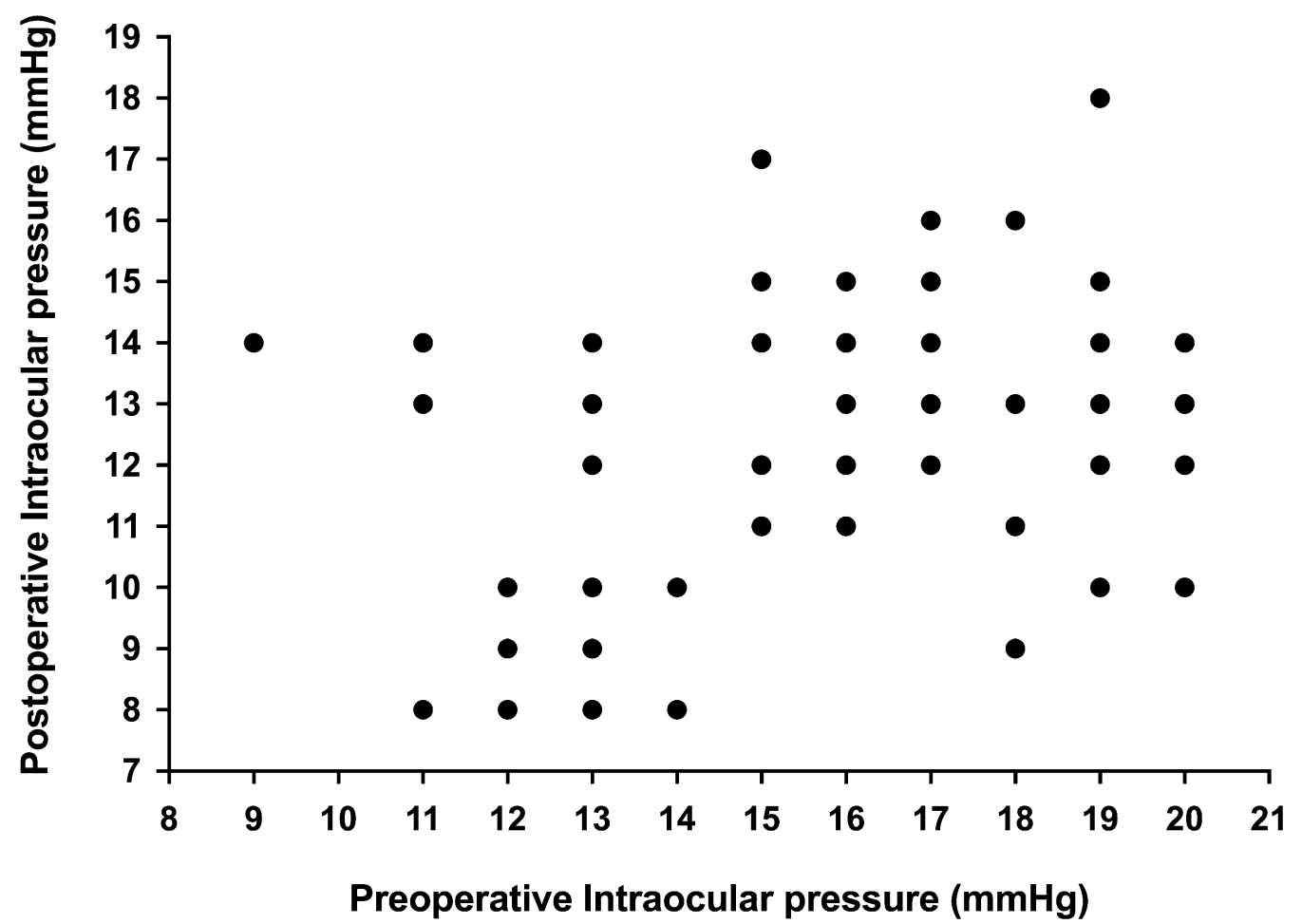

Fig. 3 Preoperative and 1-year postoperative intraocular pressure in each eye

events, endophthalmitis, hypotony, choroidal detachment, corneal decompensation, rebound iritis or stent obstruction was noted. None of the eyes underwent any further glaucoma interventions (including SLT) or surgeries within the first postoperative year.

\section{DISCUSSION}

The favorable safety and efficacy of the iStent inject trabecular micro-bypass stents has been well documented, with most evidence focusing on patients with mild-to-moderate POAG [19-27]. To the best of our knowledge, the present study is the first to exclusively examine the utility and safety of iStent inject in patients with NTG.

Considering the difficulty of treating NTG and the widely known risks of more aggressive filtering procedures [7, 34, 35], this report contributes useful information on a minimally invasive surgical approach to treat this population.
The results of our study highlighted meaningful reductions in IOP and medication use throughout 1 year following implantation of iStent inject in combination with cataract surgery. Given the well-known pattern of less dramatic IOP reduction in eyes with lower baseline IOP [34-38], it is not surprising that the IOP reduction $(3.5 \mathrm{mmHg})$ observed in these normotensive eyes was more modest than the reductions achieved in prior studies of iStent inject with phacoemulsification in predominantly POAG patients $[19,22,23,26,39]$. Although the mean IOP reduction of $3.5 \mathrm{mmHg}$ could be seen as modest, it is still noteworthy given patients' normotensive preoperative IOP and the critical role of IOP reduction even in eyes with normal pressures $[3,6]$.

The significant reduction in medication use in this study is compelling. At baseline, 39\% of eyes were on two or more glaucoma medications. In contrast, by the end of the follow-up period, the number of medications had decreased by $70 \%$, only $13 \%$ of eyes were on two or more medications, and $73 \%$ of eyes had eliminated medications entirely. This reduction 


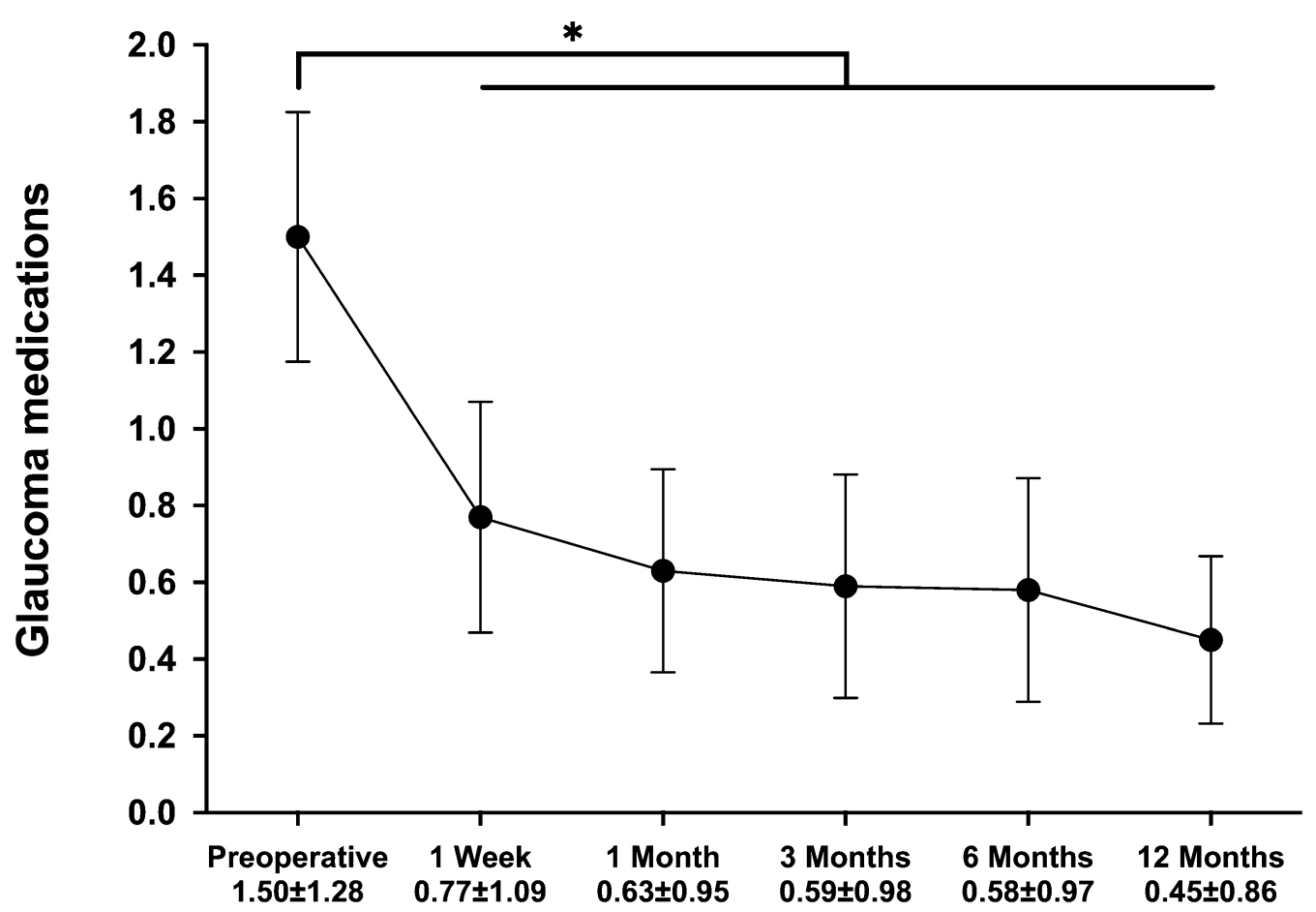

Fig. 4 Postoperative change in the number of glaucoma medications. Following cataract surgery combined with implantation of iStent inject, the mean number of medications significantly decreased and was maintained

in medication dependence is valuable in the context of the recognized difficulties with medication adherence, medication-induced ocular surface damage, local and systemic side effects, medication costs and potentially diminished quality of life $[13,15,16,20,40]$.

The safety profile in this study was highly favorable and confirms the current literature showing that adding iStent inject implantation to concomitant cataract surgery confers minimal to no additional risk beyond cataract surgery alone [41]. There was a low rate of adverse events postoperatively and no cases of severe intra- or postoperative complications. All adverse events were transient and self-limiting without sequelae. Furthermore, no patients required a secondary glaucoma intervention (laser or surgery) for continued disease progression or uncontrolled IOP in the postoperative period. The fact that these outcomes were achieved in the setting of different surgeons, sites and countries demonstrates the throughout follow-up $(p<0.001) .{ }^{*}$ Denotes statistical significance at $p<0.05$, and the error bars represent $95 \%$ confidence intervals. Mean \pm standard deviations are presented

consistency of the device's favorable safety profile regardless of setting.

This retrospective case series had certain limitations. Given the retrospective nature of the study, there was no washout phase, and strict inclusion/exclusion criteria such as those observed in clinical trials were not employed; however, it has been established that in addition to randomized control trials, real-world data are essential in health care policy-making-as the results of these studies are likely more generalizable to the real-world clinical practice [42]. In this combined procedure, the lack of a control arm limits our ability to predict to what degree the observed improvements could be attributed to the efficacy of the iStent inject alone. It is known that cataract surgery alone can lower IOP in patients with POAG; however, the evidence regarding NTG is limited. A recent study evaluating the efficacy of cataract surgery in NTG reported reductions in IOP by a mean of $2.7 \mathrm{mmHg}$ and medication use by an average of 0.82 medications at 6 


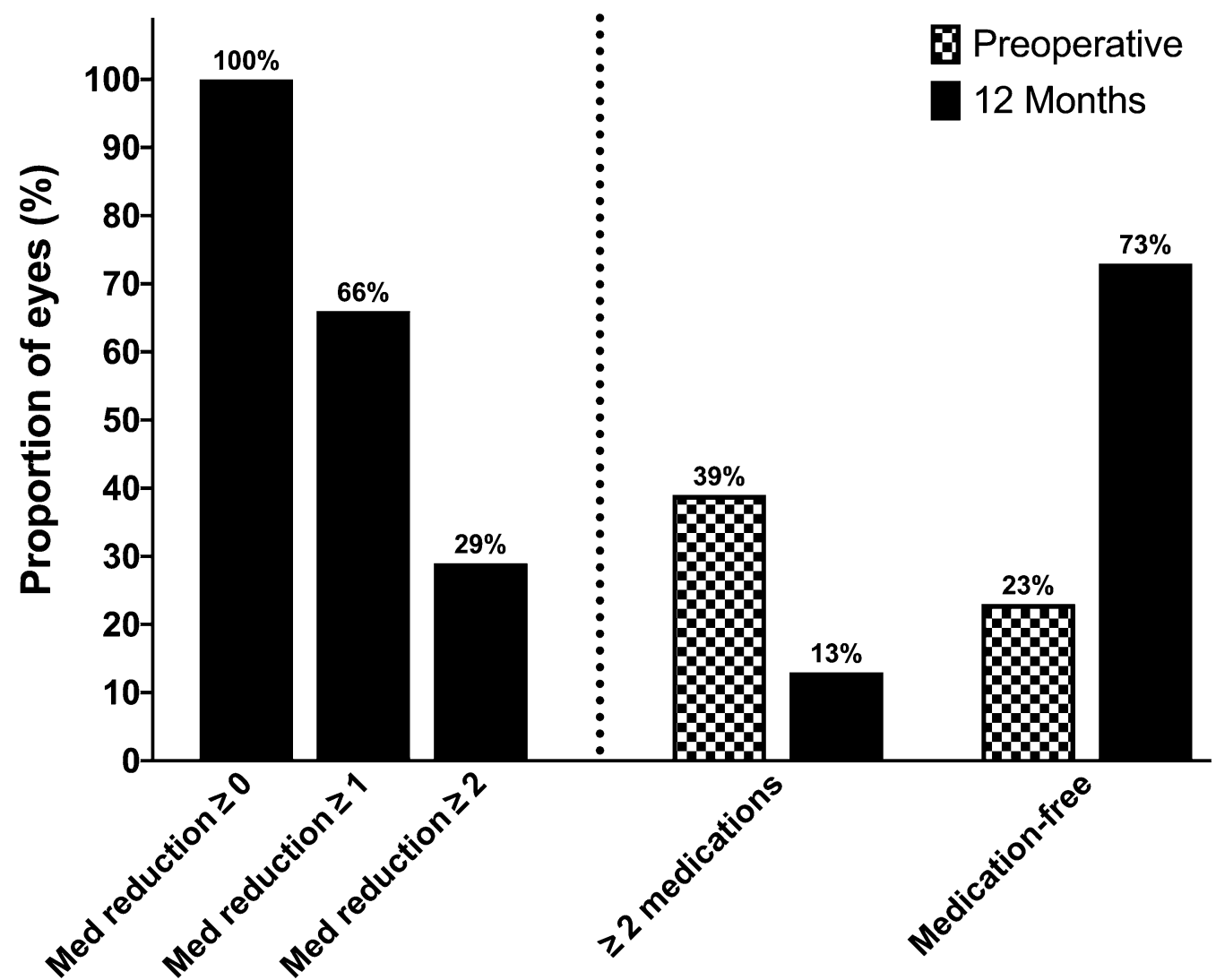

Fig. 5 Left panel (left of the dotted line) illustrates the percentage of the eyes with reductions of $\geq 0, \geq 1$ or $\geq 2$ medication(s) at 1-year follow-up. The right panel demonstrates the percentage of the eyes that are

months postoperatively [43]. A more recent study by Baek and colleagues assessed the outcomes of cataract surgery in both normal and glaucomatous eyes at 1 year and beyond [44]. A sub-analysis of the outcomes in their NTG population evidenced a mean IOP reduction of $0.78 \mathrm{mmHg}$. These results contrast to ours which evidenced reductions in IOP by a mean of $3.5 \mathrm{mmHg}$ and in medication use by an average of 1.05 medications. It is worth noting that these results should be interpreted in the context of the differences between the studies including the postoperative follow-up duration as well as the baseline clinical differences. Headto-head studies comparing the outcomes related to CE-TMS versus cataract extraction as a standalone procedure are warranted.

Despite the above-mentioned limitations, the results of this study are compelling and medication-free or on $\geq 2$ medications preoperatively (checked bars) and at 1-year follow-up (solid bars). Med: medication: reduction in the number of anti-glaucoma medications

support further investigation, particularly given the paucity of evidence regarding the use of iStent inject-and MIGS procedures more broadly-exclusively in eyes with NTG. Future prospective large-scale studies would be helpful to further elucidate the role of not only iStent inject trabecular micro-bypass, but also the category of MIGS procedures as a whole in the surgical treatment of NTG. Additionally, given the challenge of lowering IOP below the physiologic limitation of episcleral venous pressure (EVP) with MIGS procedures alone, future studies could evaluate the augmentation of MIGS procedures with other minimally invasive surgical or medical interventions for IOP reduction. 


\section{CONCLUSION}

To our knowledge, this is the first published report exclusively evaluating the outcomes of iStent inject trabecular micro-bypass with cataract surgery in NTG, a population that has not often been studied with respect to trabecular micro-bypass stents or other MIGS procedures. This report's favorable results support the use of the device in this population, in particular for patients hoping to reduce their topical medication regimen and avoid the risks associated with filtering procedures.

\section{ACKNOWLEDGEMENTS}

We thank the participants of the study.

Funding. No financial sponsorship was received for the work in this study. Rapid Service Fees were funded by Glaukos Corporation. All authors had full access to all of the data in this study and take complete responsibility for the integrity of the data and accuracy of the data analysis.

Authorship. All named authors meet the International Committee of Medical Journal Editors (ICMJE) criteria for authorship for this article, take responsibility for the integrity of the work as a whole, and have given their approval for this version to be published.

Disclosures. Paul Harasymowycz is a consultant for Allergan, Glaukos, Ivantis, J and J Vision, and Santen. Colin Clement receives travel grant, speaker, and advisory board fees from Glaukos. Ali Salimi and Michael Shiu have no relevant financial disclosures.

Compliance with Ethics Guidelines. The study was approved by the Ethics Committee of the Hôpital Maisonneuve-Rosemont in Montreal, Canada. All procedures performed were in accordance with the ethical standards of the institutional research committee and with the 1964 Helsinki Declaration and its later amendments or comparable ethical standards.
Voluntary informed consent was obtained from all participants before the surgery.

Data Availability. The datasets during and/ or analyzed during the current study are available from the corresponding author on reasonable request.

Open Access. This article is licensed under a Creative Commons Attribution-NonCommercial 4.0 International License, which permits any non-commercial use, sharing, adaptation, distribution and reproduction in any medium or format, as long as you give appropriate credit to the original author(s) and the source, provide a link to the Creative Commons licence, and indicate if changes were made. The images or other third party material in this article are included in the article's Creative Commons licence, unless indicated otherwise in a credit line to the material. If material is not included in the article's Creative Commons licence and your intended use is not permitted by statutory regulation or exceeds the permitted use, you will need to obtain permission directly from the copyright holder. To view a copy of this licence, visit http://creativecommons.org/licenses/by$\mathrm{nc} / 4.0 /$.

\section{REFERENCES}

1. Tham YC, Li X, Wong TY, Quigley HA, Aung T, Cheng CY. Global prevalence of glaucoma and projections of glaucoma burden through 2040: a systematic review and meta-analysis. Ophthalmology. 2014;121(11):2081-90.

2. Heijl A, Leske MC, Bengtsson B, Hyman L, Bengtsson B, Hussein M, et al. Reduction of intraocular pressure and glaucoma progression: results from the Early Manifest Glaucoma Trial. Arch Ophthalmol. 2002;120(10):1268-79.

3. Leske MC, Hyman L, Hussein M, Heijl A, Bengtsson B. Comparison of glaucomatous progression between untreated patients with normal-tension glaucoma and patients with therapeutically reduced intraocular pressures. The effectiveness of intraocular pressure reduction in the treatment of normal-tension glaucoma. Am J Ophthalmol. 1999;127(5):625-6. 
4. Shields MB. Normal-tension glaucoma: is it different from primary open-angle glaucoma? Curr Opin Ophthalmol. 2008;19(2):85-8.

5. Writing Committee for the Normal Tension Glaucoma Genetic Study Group of Japan Glaucoma S, Meguro A, Inoko $\mathrm{H}$, Ota M, Mizuki N, Bahram S. Genome-wide association study of normal tension glaucoma: common variants in SRBD1 and ELOVL5 contribute to disease susceptibility. Ophthalmology. 2010;117(7):1331-8 (e5).

6. Group CN-TGS. Comparison of glaucomatous progression between untreated patients with normaltension glaucoma and patients with therapeutically reduced intraocular pressures. Collaborative Normal-Tension Glaucoma Study Group. Am J Ophthalmol. 1998;126(4):487-97.

7. Gedde SJ, Herndon LW, Brandt JD, Budenz DL, Feuer WJ, Schiffman JC, et al. Postoperative complications in the Tube Versus Trabeculectomy (TVT) study during five years of follow-up. Am J Ophthalmol. 2012;153(5):804-14 (e1).

8. Saheb H, Ahmed II. Micro-invasive glaucoma surgery: current perspectives and future directions. Curr Opin Ophthalmol. 2012;23(2):96-104.

9. Ferguson TJ, Swan R, Sudhagoni R, Berdahl JP. Microbypass stent implantation with cataract extraction and endocyclophotocoagulation versus microbypass stent with cataract extraction for glaucoma. J Cataract Refract Surg. 2017;43(3): 377-82.

10. Myers JS, Masood I, Hornbeak DM, Belda JI, Auffarth $G$, Junemann A, et al. Prospective evaluation of two iStent $((\mathrm{R}))$ trabecular stents, one iStent Supra((R)) suprachoroidal stent, and postoperative prostaglandin in refractory glaucoma: 4-year outcomes. Adv Ther. 2018;35(3):395-407.

11. Shah M. Micro-invasive glaucoma surgery-an interventional glaucoma revolution. Eye Vis (Lond). 2019;6:29.

12. Prum BE Jr, Rosenberg LF, Gedde SJ, Mansberger SL, Stein JD, Moroi SE, et al. Primary open-angle glaucoma preferred practice pattern $((\mathrm{R}))$ guidelines. Ophthalmology. 2016;123(1):P41-P111.

13. Baudouin C, Labbe A, Liang H, Pauly A, BrignoleBaudouin F. Preservatives in eyedrops: the good, the bad and the ugly. Prog Retin Eye Res. 2010;29(4):312-34.

14. Joseph A, Pasquale LR. Attributes associated with adherence to glaucoma medical therapy and its effects on glaucoma outcomes: an evidence-based review and potential strategies to improve adherence. Semin Ophthalmol. 2017;32(1):86-90.
15. Leung EW, Medeiros FA, Weinreb RN. Prevalence of ocular surface disease in glaucoma patients. J Glaucoma. 2008;17(5):350-5.

16. Patel V, Ahmed I, Podbielski D, Falvey H, Murray J, Goeree R. Cost-effectiveness analysis of standalone trabecular micro-bypass stents in patients with mild-to-moderate open-angle glaucoma in Canada. J Med Econ. 2019;22(4):390-401.

17. Robin AL, Covert D. Does adjunctive glaucoma therapy affect adherence to the initial primary therapy? Ophthalmology. 2005;112(5):863-8.

18. Muniesa MJ, Ezpeleta J, Benitez I. Fluctuations of the intraocular pressure in medically versus surgically treated glaucoma patients by a contact lens sensor. Am J Ophthalmol. 2019;203:1-11.

19. Arriola-Villalobos P, Martinez-de-la-Casa JM, DiazValle D, Morales-Fernandez L, Fernandez-Perez C, Garcia-Feijoo J. Glaukos iStent inject(R) trabecular micro-bypass implantation associated with cataract surgery in patients with coexisting cataract and open-angle glaucoma or ocular hypertension: a long-term study. J Ophthalmol. 2016;2016: 1056573.

20. Berdahl J, Voskanyan L, Myers JS, Hornbeak DM, Giamporcaro JE, Katz LJ, et al. Implantation of two second-generation trabecular micro-bypass stents and topical travoprost in open-angle glaucoma not controlled on two preoperative medications: 18-month follow-up. Clin Exp Ophthalmol. 2017;45(8):797-802.

21. Fea AM, Belda JI, Rekas M, Junemann A, Chang L, Pablo L, et al. Prospective unmasked randomized evaluation of the iStent inject ((R)) versus two ocular hypotensive agents in patients with primary openangle glaucoma. Clin Ophthalmol. 2014;8:875-82.

22. Guedes RAP, Gravina DM, Lake JC, Guedes VMP, Chaoubah A. Intermediate results of iStent or iStent inject implantation combined with cataract surgery in a real-world setting: a longitudinal retrospective study. Ophthalmol Ther. 2019;8(1):87-100.

23. Hengerer FH, Auffarth GU, Riffel C, Conrad-Hengerer I. Prospective, non-randomized, 36-month study of second-generation trabecular micro-bypass stents with phacoemulsification in eyes with various types of glaucoma. Ophthalmol Ther. 2018;7(2):405-15.

24. Klamann MK, Gonnermann J, Pahlitzsch M, Maier $\mathrm{AK}$, Joussen AM, Torun $\mathrm{N}$, et al. iStent inject in phakic open angle glaucoma. Graefes Arch Clin Exp Ophthalmol. 2015;253(6):941-7.

25. Lindstrom R, Lewis R, Hornbeak DM, Voskanyan L, Giamporcaro JE, Hovanesian J, et al. outcomes 
following implantation of two second-generation trabecular micro-bypass stents in patients with open-angle glaucoma on one medication: 18-month follow-up. Adv Ther. 2016;33(11): 2082-90.

26. Salimi A, Lapointe J, Harasymowycz P. One-year outcomes of second-generation trabecular microbypass stents (iStent Inject) implantation with cataract surgery in different glaucoma subtypes and severities. Ophthalmol Ther. 2019;8(4):563-75.

27. Voskanyan L, Garcia-Feijoo J, Belda JI, Fea A, Junemann A, Baudouin C, et al. Prospective, unmasked evaluation of the iStent $(\mathrm{R})$ inject system for open-angle glaucoma: synergy trial. Adv Ther. 2014;31(2):189-201.

28. Hann CR, Fautsch MP. Preferential fluid flow in the human trabecular meshwork near collector channels. Invest Ophthalmol Vis Sci. 2009;50(4):1692-7.

29. Bostan C, Harasymowycz P. Episcleral venous outflow: a potential outcome marker for iStent surgery. J Glaucoma. 2017;26(12):1114-9.

30. Salimi A, Kovalyuk N, Harasymowycz PJ. Tube shunt revision with excision of fibrotic capsule using Mitomycin C with and without Ologen-a Collagen matrix implant: A 3-year follow-up study. J Glaucoma. 2019;28(11):989-96.

31. Heuer D, Barton K, Grehn F, Shaarawy T, Sherwood $\mathrm{M}$. Guidelines on design and reporting of glaucoma surgical trials. Amsterdam, The Netherlands: Kugler Publications; 2008.

32. Hodapp E, Parrish RK, Anderson DR. Clinical decisions in glaucoma. Missouri: Mosby Incorporated; 1993.

33. Salimi A, Winter A, Li C, Harasymowycz P, Saheb H. Effect of topical corticosteroids on early postoperative intraocular pressure following combined cataract and trabecular microbypass surgery. J Ocul Pharmacol Ther. 2019;35(7):413-20.

34. Jampel HD, Musch DC, Gillespie BW, Lichter PR, Wright MM, Guire KE, et al. Perioperative complications of trabeculectomy in the collaborative initial glaucoma treatment study (CIGTS). Am J Ophthalmol. 2005;140(1):16-22.

35. Rulli E, Biagioli E, Riva I, Gambirasio G, De Simone I, Floriani I, et al. Efficacy and safety of trabeculectomy vs nonpenetrating surgical procedures: a systematic review and meta-analysis. JAMA Ophthalmol. 2013;131(12):1573-82.

36. Ferguson T, Swan R, Ibach M, Schweitzer J, Sudhagoni R, Berdahl JP. Evaluation of a trabecular microbypass stent with cataract extraction in severe primary open-angle glaucoma. J Glaucoma. 2018;27(1):71-6.

37. Ferguson TJ, Ibach M, Schweitzer J, Karpuk K, Stephens JD, Sudhagoni R, et al. Trabecular microbypass stent implantation in pseudophakic eyes with open-angle glaucoma: long-term results. J Cataract Refract Surg. 2019;45(4):414-20.

38. Poley BJ, Lindstrom RL, Samuelson TW. Long-term effects of phacoemulsification with intraocular lens implantation in normotensive and ocular hypertensive eyes. J Cataract Refract Surg. 2008;34(5): 735-42.

39. Clement CI, Howes F, Ioannidis AS, Shiu M, Manning D. One-year outcomes following implantation of second-generation trabecular micro-bypass stents in conjunction with cataract surgery for various types of glaucoma or ocular hypertension: multicenter, multi-surgeon study. Clin Ophthalmol. 2019;13:491-9.

40. Fechtner RD, Voskanyan L, Vold SD, Tetz M, Auffarth G, Masood I, et al. Five-year, prospective, randomized, multi-surgeon trial of two trabecular bypass stents versus prostaglandin for newly diagnosed open-angle glaucoma. Ophthalmol Glaucoma. 2019;2(3):156-66.

41. Samuelson TW, Sarkisian SR Jr, Lubeck DM, Stiles MC, Duh YJ, Romo EA, et al. Prospective, randomized, controlled pivotal trial of an $\mathrm{Ab}$ interno implanted trabecular micro-bypass in primary open-angle glaucoma and cataract: two-year results. Ophthalmology. 2019;126(6):811-21.

42. Garrison LP Jr, Neumann PJ, Erickson P, Marshall D, Mullins CD. Using real-world data for coverage and payment decisions: the ISPOR Real-World Data Task Force report. Value Health. 2007;10(5):326-35.

43. Lee W, Bae HW, Kim CY, Seong GJ. The change of anterior segment parameters after cataract surgery in normal-tension glaucoma. Int J Ophthalmol. 2017;10(8):1239-45.

44. Baek SU, Kwon S, Park IW, Suh W. Effect of phacoemulsification on intraocular pressure in healthy subjects and glaucoma patients. J Korean Med Sci. 2019;34(6):e47. 